\title{
Alteration of coenzyme specificity of malate dehydrogenase from Streptomyces coelicolor A3(2) by site-directed mutagenesis
}

Y.D. Ge, P. Song, Z.Y. Cao, P. Wang and G.P. Zhu

Institute of Molecular Biology and Biotechnology, Key Laboratory of Molecular Evolution and Biodiversity, Key Laboratory of the Biotic Environment and Ecological Safety in Anhui Province, Anhui Normal University, Wuhu, Anhui, China

Corresponding author: G.P. Zhu

E-mail: gpz1996@yahoo.com

Genet. Mol. Res. 13 (3): 5758-5766 (2014)

Received May 29, 2013

Accepted October 23, 2014

Published July 29, 2014

DOI http://dx.doi.org/10.4238/2014.July.29.3

\begin{abstract}
We describe here for the first time the alteration of coenzyme specificity of malate dehydrogenase (MDH) from Streptomyces coelicolor A3(2) (ScMDH). In the present study, we replaced four amino acid residues in the Rossmann fold $(\beta \mathrm{B}-\alpha \mathrm{C})$ region of NADH-dependent $S c \mathrm{MDH}$ by site-directed mutagenesis with those of NADPH-dependent MDH (Glu42Gly, Ile43Ser, Pro45Arg, and Ala46Ser). The coenzyme specificity of the mutant enzyme (ScMDH-T4) was examined. Coenzyme specificity of ScMDH-T4 was shifted 2231.3 -fold toward NADPH using $k_{\text {cat }} / K_{\mathrm{m}}$ coenzyme as the measurement of coenzyme specificity. Accordingly, the effect of the replacements on coenzyme specificity is discussed. Our work provides further insight into the coenzyme specificity of $S c \mathrm{MDH}$.
\end{abstract}

Key words: Malate dehydrogenase; Coenzyme specificity; Streptomyces coelicolor; Site-directed mutagenesis 


\section{INTRODUCTION}

Malate dehydrogenase (MDH) catalyzes the reversible conversion of malate to oxalacetate using $\mathrm{NAD}^{+}$or $\mathrm{NADP}^{+}$as the coenzyme (Madern et al., 2004; Hara et al., 2006). It plays crucial roles in many metabolic pathways, including the tricarboxylic acid cycle, amino acid synthesis, gluconeogenesis, maintenance of the oxidation/reduction balance and metabolic stress (Goward and Nicholls, 1994; Lee et al., 2009; Liu et al., 2013; Menckhoff et al., 2013).

$\mathrm{MDH}$ can be divided into $\mathrm{NAD}^{+}$-dependent $\mathrm{MDH}$ (NAD-MDH) (EC 1.1.1.37) and $\mathrm{NADP}^{+}$-dependent MDH (NADP-MDH) (EC 1.1.1.82) (Lee et al., 2009), according to preference for cofactors. Most bacterial and archaeal MDHs are NAD-MDH. Eukaryotic MDH isoforms are all NAD-MDH, including mitochondrial MDH (mMDH), cytosolic MDH (cMDH), glyoxysomal MDH (gMDH), and chloroplastic NAD-MDH (cnMDH), except for chloroplastic NADP-MDH (chMDH), which is required for the transfer of reducing equivalents from chloroplast stroma to cytosol (Hara et al., 2006).

There are various $\mathrm{NAD}(\mathrm{P})(\mathrm{H})$-dependent oxidoreductases with a Rossmann fold $(\beta \mathrm{B}-$ $\alpha \mathrm{C})$ for dinucleotide binding. The coenzyme-binding domains in the dehydrogenase enzyme family have been thoroughly investigated, revealing a conserved $\beta \mathrm{B}-\alpha \mathrm{C}$ motif of the Rossmann fold (Rossmann et al., 1974; Lesk, 1995). The ability of the dehydrogenases to discriminate against $\mathrm{NADP}(\mathrm{H})$ lies in the amino acid sequence of this $\beta \mathrm{B}-\alpha \mathrm{C}$ motif, which has been predicted to be a principal determinant for coenzyme specificity. By replacing the corresponding region of $N A D(H)-$ MDH from Thermus flavus (TfMDH) in the Rossmann fold with those of NADP(H)-MDH, tMDH was successfully altered from an NAD-dependent enzyme to NADP-dependent enzyme, showing that the preference of mutant $T f \mathrm{MDH}$ for NADPH over NADH is mainly due to the combined recognition of the 2'-phosphate group of NADPH by four amino acid residues (Gly41, Ser42, Arg44, and Ser45) from NADP(H)-MDH in the Rossmann fold (Tomita et al., 2006b).

Several attempts to alter the coenzyme specificity have been made for several dehydrogenases, and complete reversal was accomplished in xylitol dehydrogenase from Pichia stipitis and Saccharomyces cerevisiae (Watanabe et al., 2005; Krahulec et al., 2012), alcohol dehydrogenase from Rana perezi (Rosell et al., 2003) and lactate dehydrogenases from T. flavus (Tomita et al., 2006a). However, the information on alteration of coenzyme specificity in bacterial MDHs is scarce, except for TfMDH.

Streptomyces (multicellular prokaryotes) are Gram-positive soil bacteria that carry out complex morphological differentiation in the formation of filamentous mycelium-like fungi (eukaryotes), an example of convergent evolution caused by adaptation to similar ecological niches. Also, they are model prokaryotes for the study of evolution and secondary metabolism. Previously, MDH from Streptomyces coelicolor A3(2) (ScMDH) was overexpressed in Escherichia coli and its biochemical properties were identified in detail (Ge et al., 2010). ScMDH is an $\mathrm{NAD}(\mathrm{H})$-dependent thermostable enzyme composed of two identical subunits. However, the information on the coenzyme specificity evolution of $S c \mathrm{MDH}$ is very limited.

To get a preliminary understanding of the molecular mechanism of coenzyme evolution relationship between NADP-MDH and NAD-MDH, in this study, we introduced four point mutations (Glu42Gly, Ile43Ser, Pro45Arg, and Ala46Ser) by site-directed mutagenesis into ScMDH and examined the coenzyme specificity of the resulting enzyme. The amino acid substitution in the Rossmann fold markedly shifted the coenzyme specificity of $S c \mathrm{MDH}$ toward NADPH. To our knowledge, this is the first report on the coenzyme specificity of MDH from Streptomyces. 


\section{MATERIAL AND METHODS}

\section{Strains and reagents}

E. coli DH5 $\alpha$ was used as the host for genetic cloning. E. coli Rosetta (DE3) and plasmid pET-28b $(+)$ were used for protein expression. PureYield ${ }^{\mathrm{TM}}$ Plasmid Miniprep System kit and Wizard ${ }^{\circledR}$ SV Gel and PCR Clean-Up System kit were from Promega (Madison, WI, USA). Restriction enzymes were from Sangon Biotech (Shanghai, China). All PCR reactions were performed with PrimeSTAR ${ }^{\circledR}$ HS DNA Polymerase (TaKaRa, Japan). Proteins were purified using BD TALON ${ }^{\circledR}$ xTractor Buffer and TALON Metal Affinity Resins (Clontech, Beijing, China). Oxaloacetate (OAA) was from Sigma-Aldrich (Shanghai, China). NAD(P) ${ }^{+}$ and NAD(P)H were from Bio Basic Inc. (Markham, Canada). Primers were synthesized by GenScript Co., Ltd. (Nanjing, China). The ScMDH gene was cloned from the recombinant plasmid pScMDH (Ge et al., 2010).

\section{Primer design}

The synthetic oligonucleotide primers were designed with the aid of the Primer Premier 5.0 software (Premier, Canada) and were based on the recombinant plasmid pScMDH. Four oligonucleotides were used: two flanking primers were positioned upstream (U) and downstream (D) of the mutation site, and two mutagenic primers, forward mutagenic (FM) and reverse mutagenic (RM) with a 20-bp overlap between adjacent fragments. The mutation site was located in the middle of the mutagenic primers. T4 FM and T4 RM were used as mutagenic primers for the construction of mutant enzyme having the Glu42Gly, Ile43Ser, Pro45Arg and Ala46Ser exchanges; the mutant was named ScMDH-T4. The primer sequences used in this study are shown in Table 1.

\begin{tabular}{ll}
\multicolumn{2}{c}{ Table 1. Mutagenic and flanking primers. } \\
\hline Primers & Sequence $\left(5^{\prime} \text { to } 3^{\prime}\right)^{\mathrm{a}}$ \\
\hline $\mathrm{U}$ & GAGACACCGGCATACTCTGCGACAT \\
D & CGCCCCTCGAGTCAGATGAGGCCGAGACCGCGC \\
T4 FM & CTTGAGactCGGGTGcTCCCCAGGAGGGCAGCTTGACC \\
T4 RM & gGAgCACCCgGagtCTCAAGGCCGCCGAGGGCACCGCGATGGA \\
\hline
\end{tabular}

${ }^{a}$ Nucleotide substitution is shown in boldface and lower case; underlined nucleotides (CTCGAG) represent the restriction site for XhoI enzyme.

\section{Site-directed mutagenesis}

Purification of recombinant plasmid $\mathrm{pScMDH}$ DNA was performed using the Wizard ${ }^{\circledR}$ purification kit. Site-directed mutations were introduced into the ScMDH gene by Simionatto's method (Simionatto et al., 2009). It was carried out according to a two-step PCR procedure to replace the mutated codons using an overlap extension-PCR method. Briefly, in the first step, two simultaneous PCRs were performed. One reaction was performed with a primer pair that included the $\mathrm{U}$ primer and the RM primer; the other reaction contained the $\mathrm{D}$ primer and the FM primer. PCRs were carried out using $1 \mathrm{ng}$ recombinant plasmid pScMDH DNA, $40 \mathrm{nM}$ 
dNTPs, $10 \mathrm{pM}$ of each primer, 1.3 U PrimeSTAR ${ }^{\circledR}$ HS DNA Polymerase, and $1 \mathrm{X}$ reaction buffer in a $50-\mu \mathrm{L}$ reaction volume. The amplification conditions were $3 \mathrm{~min}$ at $98^{\circ} \mathrm{C}$ followed by 30 cycles of $20 \mathrm{~s}$ at $98^{\circ} \mathrm{C}, 25 \mathrm{~s}$ at $65^{\circ} \mathrm{C}$ and $60 \mathrm{~s}$ at $72^{\circ} \mathrm{C}$ and a final extension of $5 \mathrm{~min}$ at $72^{\circ} \mathrm{C}$. PCR products were analyzed by $1 \%$ agarose gel electrophoresis and gel-purified using the Wiz$\operatorname{ard}^{\circledR}$ SV Gel and PCR Clean-Up System kit, according to manufacturer instructions. To obtain full-length mutated fragments, the two products from the first PCR were mixed at equimolar concentrations (approximately $1.5 \mathrm{pM}$ ) and used as templates during the second PCR, with the $\mathrm{U}$ and D primers. The reaction was performed using the same conditions as the first PCR except that the annealing temperature and extension time were $55^{\circ} \mathrm{C}$ and $100 \mathrm{~s}$, respectively. This overlap extension-PCR yielded a full-length DNA fragment, which was gel-purified before cloning. The PCR product was digested with $\mathrm{NdeI}$ and $\mathrm{XhoI}$ and ligated into pET-28b(+)His-tag expression vector. The mutated $S c \mathrm{MDH}$ gene was confirmed by DNA sequencing. After confirming the presence of the mutated codons, the mutated plasmids were transformed into E. coli Rosetta cells (DE3). The recombinant mutant $S c \mathrm{MDH}-\mathrm{T} 4$ was expressed and purified as previously described for the wild-type $S c \mathrm{MDH}$ (Ge et al., 2010).

\section{Kinetic assays and data processing}

Wild-type $S c \mathrm{MDH}$ and mutant $S c \mathrm{MDH}-\mathrm{T} 4$ activity studies were performed in $100 \mathrm{~mm}$ Tris- $\mathrm{HCl}, \mathrm{pH} 8.5$, buffer according to the methods previously reported (Ge et al., 2010). The reaction was monitored by measuring the decrease in absorbance of $\mathrm{NAD}(\mathrm{P}) \mathrm{H}$ at $340 \mathrm{~nm}$ with a thermostated Cary 300 UV-Vis spectrophotometer (Varian, Shanghai, China) using a molar extinction coefficient of $6220 \mathrm{M}^{-1} \cdot \mathrm{cm}^{-1}$. One unit (U) of enzyme activity was defined as the amount of enzyme required to produce $1 \mu \mathrm{mol} \operatorname{NAD}(\mathrm{P}) \mathrm{H} / \mathrm{min}$ under the assay conditions $\left(30^{\circ} \mathrm{C}\right)$. All kinetic parameters were obtained from at least triplicate measurements. Protein concentrations were determined using a Bio-Rad protein assay kit with bovine serum albumin as standard.

\section{Molecular simulation of wild-type $S c \mathrm{MDH}$ complex with NADH}

Molecular simulation was carried out with the PyMOL-molecular visualization system and the SWISS-MODEL Workspace web tool (http://swissmodel.expasy.org/workspace/ index.php?func=modelling_simple1). The crystal structure of TfMDH complex with NADH (PDB accession No. 1BMD) was used as an initial model of molecular simulation. The predicted three-dimensional structure of wild-type $S c \mathrm{MDH}$ complex with NADH was modeled.

\section{RESULTS}

\section{Coenzyme specificity of wild-type $S c$ MDH and mutant ScMDH-T4}

The steady-state kinetic parameters of wild-type $S c \mathrm{MDH}$ and mutant $S c \mathrm{MDH}-\mathrm{T} 4$ were determined with both coenzymes (NADH and NADPH) in the presence of OAA. The mutant enzyme was assumed to have the same kinetic mechanism as the wild-type enzyme. As shown in Table 2, wild-type $S c \mathrm{MDH}$ showed a significant preference for NADH over NADPH with 13.4-fold decreased $K_{\mathrm{m}}$ value for NADH and a 15 -fold larger $k_{\text {cat }}$ value for the reaction. As a consequence, wild-type ScMDH showed 218.7-fold coenzyme specificity for NADH over NADPH 
taking $k_{\text {cat }} / K_{\mathrm{m}}$ coenzyme as a measure. The four-amino-acid replacement in ScMDH-T4 caused 4.8fold increase in the $K_{\mathrm{m}}$ value for NADH, but the $k_{\text {cat }}$ value was not decreased too much. As for the NADPH-dependent reaction, the replacement decreased the $K_{\mathrm{m}}$ value for NADPH by 8.2-fold. As a consequence, the $K_{\mathrm{m}}$ value for NADH was about 3-fold larger than that for NADPH in ScMDHT4. The most dramatic change in the parameters by the amino acid replacement was found in the $k_{\text {cat }}$ values. As described above, the $k_{\text {cat }}$ value in the NADH-dependent reaction decreased a little. However, the replacement increased the $k_{\text {cat }}$ value for NADPH oxidation by 47.5 -fold, resulting in a 3.5-fold larger $k_{\text {cat }}$ value in the NADPH-dependent reaction as compared with that in the NADHdependent reaction. As a result, when $k_{\mathrm{cat}} / K_{\mathrm{m}}^{\text {coenzyme }}$ value was again considered as a measure of the catalytic efficiency of the enzyme, ScMDH-T4 increased 423.7-fold for NADPH and decreased 5.3-fold for NADH, compared with the case of wild-type $S c \mathrm{MDH}$. When a preference for NADH over NADPH was defined by $\left(k_{\text {cat }} / K_{\mathrm{m}}^{\mathrm{NADH}}\right) /\left(k_{\mathrm{cat}} / K_{\mathrm{m}}^{\mathrm{NADPH}}\right)$ (Tomita et al., 2006b; Andreadeli et al., 2008), it showed 218.67 for wild-type $S c \mathrm{MDH}$ and 0.098 for $S c \mathrm{MDH}-\mathrm{T} 4$ (Table 3). This means that the four-amino-acid replacement shifted the coenzyme specificity of ScMDH 2231.3-fold toward NADPH, which is significantly larger than the shift in TfMDH alteration of coenzyme specificity, namely 1562.6-fold (Tomita et al., 2006b), as shown in Table 3.

Table 2. Kinetic parameters of wild-type and mutant $S c \mathrm{MDH}$.
\begin{tabular}{llrrr}
\hline Enzyme & Coenzyme & $K_{\mathrm{m}}(\mu \mathrm{M})$ & $k_{\text {cat }}\left(\mathrm{s}^{-1}\right)$ & $k_{\text {cat }} / K_{\mathrm{m}}\left(\mathrm{s}^{-1} \cdot \mu \mathrm{M}^{-1}\right)$ \\
\hline Wild-type $S c \mathrm{MDH}$ & $\mathrm{NADH}$ & 82.58 & 542.00 & 6.56 \\
\multirow{2}{*}{$S c$ MDH-T4 } & NADPH & 1106.48 & 36.07 & 0.03 \\
& $\mathrm{NADH}$ & 399.72 & 494.54 & 1.24 \\
& NADPH & 134.66 & 1711.54 & 12.71 \\
\hline
\end{tabular}

Table 3. Comparison of the degree of alteration of coenzyme specificity toward NADPH in ScMDH-T4 with the mutant MDH from Thermus flavus.

\begin{tabular}{|c|c|c|c|c|}
\hline \multirow[t]{2}{*}{ Enzyme } & \multicolumn{2}{|c|}{$k_{\mathrm{cat}} / K_{\mathrm{m}}\left(\mathrm{s}^{-1} \cdot \mu \mathrm{M}^{-1}\right)$} & \multirow[t]{2}{*}{ Preference (A)/(B) } & \multirow[t]{2}{*}{ Degree of alteration } \\
\hline & NADH (A) & NADPH (B) & & \\
\hline Wild-type $S c \mathrm{MDH}$ & 6.56 & 0.03 & 218.67 & 2231.3 \\
\hline ScMDH-T4 & 1.24 & 12.71 & 0.098 & \\
\hline TfMDH & 61.1 & 1.7 & 35.94 & 1562.6 \\
\hline TfMDH-EX4 & 1.1 & 48.3 & 0.023 & \\
\hline
\end{tabular}

\section{In silico simulation of wild-type $S c \mathrm{MDH}$ structure}

To understand the reason for the shift of the coenzyme specificity of $S c \mathrm{MDH}$ in detail, structural information is necessary. Although the high resolution three-dimensional structure of $S c \mathrm{MDH}$ has not yet been determined, the amino acid sequence of $S c \mathrm{MDH}$ is very similar to that of TfMDH, with $65 \%$ identity, and there is only one gap, located at a position far from the active site and the coenzyme binding site, between the two MDHs. Furthermore, they have high similar amino acid sequence in the Rossmann fold $(\beta \mathrm{B}-\alpha \mathrm{C})$ for dinucleotide binding (Figure 1). Hence, we performed in silico modeling of the three-dimensional structure of wildtype $S c \mathrm{MDH}$ using the crystal structure of TfMDH complex with NADH (PDB entry 1BMD) as the initial template structure (Figure 2A). As can be seen in Figure 2B, simulated ScMDH structure almost superimposes with the TfMDH binary complex with NADH. 


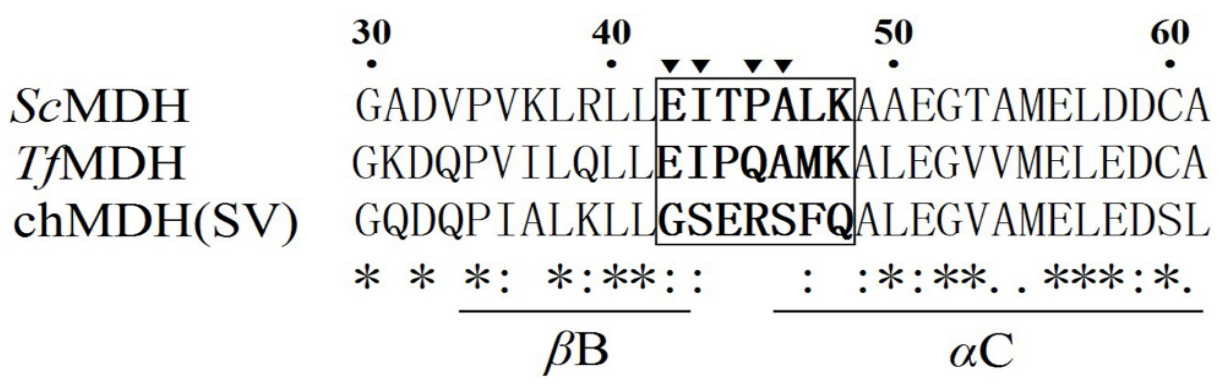

Figure 1. Alignment of the amino acid sequences in the region between $\beta$-strand B and $\alpha$-helix C. ScMDH, TfMDH, and chMDH(SV) represent MDHs from Streptomyces coelicolor A3(2), Thermus flavus and Sorghum vulgare chloroplasts, respectively. The replaced amino acid residues in mutant $S c \mathrm{MDH}$ was indicated by inverted dark triangle. The alignment was done using the CLUSTAL W 2.0. The residues in the $\beta B-\alpha C$ loop are boxed and the location of $\beta$-strand $\mathrm{B}$ and $\alpha$-helix $\mathrm{C}$ are marked below the sequences. The amino acid sequence numbers above the sequences are those for $\mathrm{ScMDH}$.
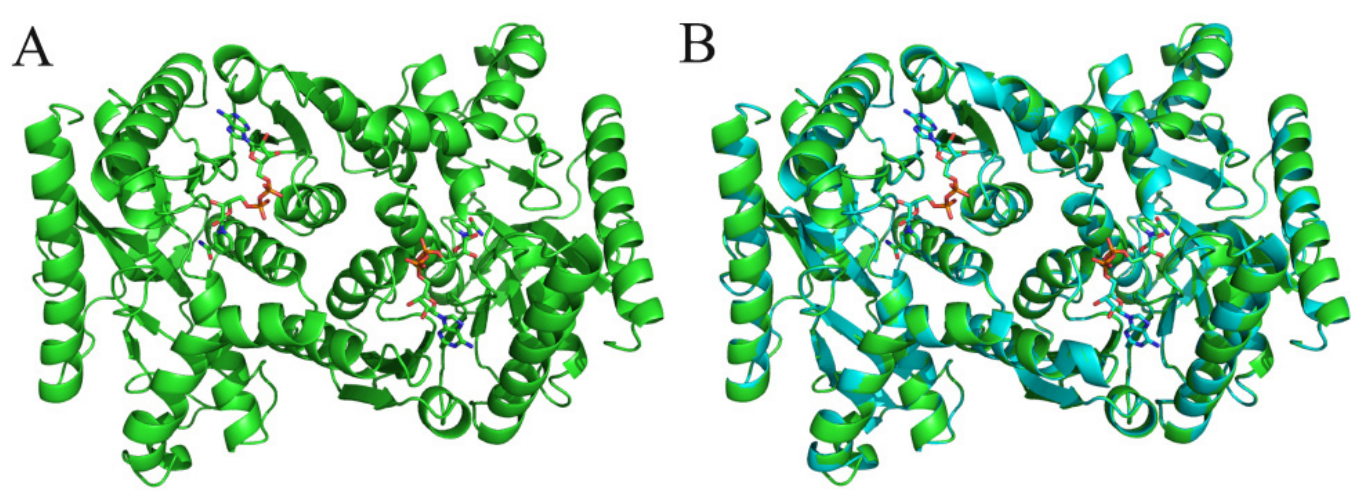

Figure 2. Comparison of the ribbon diagram between $S c \mathrm{MDH}$ and $T f \mathrm{MDH}$. A. Simulated ScMDH dimer threedimensional structure binary complex with NADH; B. stereoview of a superposition of simulated ScMDH threedimensional structure (green) on TfMDH (light blue) binary complex with NADH.

\section{DISCUSSION}

Previous studies had shown that the preference of mutant TfMDH for NADPH over NADH is mainly determined by four amino acid residues (Gly41, Ser42, Arg44, and Ser45), which corresponded to the amino acid residues in NADPH-dependent chloroplast MDH's Rossmann fold ( $\beta \mathrm{B}-\alpha \mathrm{C})$ (Tomita et al., 2006b). The earlier investigations suggested that the hydrogen-bond interactions between two Ser residues (Ser42 and Ser45) and the 2'-phosphate of NADPH are the crucial factors in the high affinity of mutant TfMDH for NADPH, where Gly41 allows the 2'-phosphate group of NADPH to enter the binding site (Tomita et al., 2006b). In addition, Arg44 was presumed to form an additional electrostatic interaction with the 2'-phosphate of NADPH to stabilize the binding in mutant TfMDH (Tomita et al., 2006b).

In this study, we succeeded in altering the coenzyme specificity of MDH from Streptomyces coelicolor $\mathrm{A} 3(2)$ by substituting the Rossmann fold $(\beta \mathrm{B}-\alpha \mathrm{C})$ with the corresponding 
four amino acids in NADPH-dependent chloroplast MDH: Glu42Gly, Ile43Ser, Pro45Arg, Ala46Ser. The replacement shifted the coenzyme specificity 2231.3-fold from NADH to NADPH in the $S c \mathrm{MDH}-\mathrm{T} 4$ mutant. This result suggested that the preference of $S c \mathrm{MDH}-\mathrm{T} 4$ for NADPH over NADH was mainly due to the four-amino-acid replacement from NADP(H)$\mathrm{MDH}$ in the Rossmann fold $(\beta \mathrm{B}-\alpha \mathrm{C})$.

Structure simulation revealed that the predicted three-dimensional structure of wildtype $S c \mathrm{MDH}$ was essentially the same as that of TfMDH (Figure 2B). The high homology in three-dimensional structure coupled with the high similar amino acid sequence in the Rossmann fold suggested that $S c \mathrm{MDH}$ had a similar coenzyme catalytic mechanism as TfMDH.

In comparison to the results in the four-amino-acid mutant TfMDH, in ScMDH-T4, the reversal of coenzyme specificity from NADH to NADPH was also achieved with the concerted mutation of four residues (Glu42Gly, Ile43Ser, Pro45Arg, Ala46Ser). It was reported that the negatively charged residue is one of the most principal determinants for the preference for NADH over NADPH, and the reasons for the coenzyme specificity change of MDH lied in the side-chain differences (Tomita et al., 2006b). Thus, the high affinity of ScMDH-T4 for NADPH over NADH could be speculated on the basis of these possible effects. First, due to electrostatic repulsion along with steric hindrance, the carboxyl group of Glu41 prevents the 2'-phosphate group of NADPH from binding. In ScMDH-T4, due to the Glu42Gly mutation, the hydrogen-bonds between the carboxyl group of Glu42 and the O2' and O3' atoms of the adenine ribose moiety of NADH are removed, and the 2'-phosphate group of the adenine ribose in NADPH can enter and occupy the position vacated by the mutation. Second, in ScMDH-T4, Ser43 and Ser46 are located too far from the O2' and O3' atoms of the adenine ribose moiety of NADH to form hydrogen-bonds according to the crystal structure of the mutant TfMDH complex with NADPH (Tomita et al., 2006b). On the other hand, OP1A of NADPH is hydrogen-bonded to the side-chain of the Ser residues replaced at positions 43 and 46. The hydrogen-bond interactions between these Ser residues, 43 and 46, and the 2'-phosphate of NADPH may be the crucial factors in the tight affinity of ScMDH-T4 for NADPH; in addition, the importance of the corresponding Arg residues in NADPH specificity has been shown in mutant TfMDH and other NADPH-dependent dehydrogenases (Scrutton et al., 1990; Levy et al., 1996; Schepens et al., 2000). In this study, we speculated that the substitution of Pro45Arg in ScMDH-T4 enabled the Arg45 to form an additional electrostatic interaction with the 2'-phosphate of NADPH to stabilize the binding between the enzyme and the coenzyme.

Notably, phylogenetic analysis indicates that the NADPH-dependent MDHs seem to have descended from NADH-dependent progenitors during MDH gene evolution (Ocheretina et al., 2000; Schnarrenberger and Martin, 2002). However, no conclusive experimental evidence has been provided to support this theory up to now. Site-directed mutagenesis experiments have shown that the coenzyme specificity of MDH is determined by a limited number of amino acid residues (Tomita et al., 2005, 2006b), and that the coenzyme specificity of some dehydrogenases could be shifted by alteration of a few specific amino acids in the enzyme to adapt to the environment during the enzyme's evolution (Zhu et al., 2005). In the present study, we altered the coenzyme specificity of MDH from a Gram-positive bacterium $S$. coelicolor A3(2). Together with the study of coenzyme specificity alteration in $T f \mathrm{MDH}$, our investigation of $S c \mathrm{MDH}$ may give new experimental evidence for the coenzyme specificity evolution theory of MDH described above. That is to say, the coenzyme specificity of MDH can be altered just by a few amino acids substitution at crucial sites of the enzyme. Moreover, 
amino acid substitution is not a difficult event during the long evolutionary history of MDH. Thus, it can be supposed that the coenzyme specificity of an NADPH-MDH ancestor was shifted from NADH toward NADPH by the mutation of just a few amino acids in response to the external pressures from living environment.

In summary, our study describes for the first time the alteration of coenzyme specificity of MDH from Streptomyces. The results showed that $S c \mathrm{MDH}$ was successfully altered from an NADH-dependent to an NADPH-dependent enzyme by a four-amino-acid substitution. This work provides the further insight into the universality of coenzyme conversion and the coenzyme specificity of $S c \mathrm{MDH}$. The crystal structures of $S c \mathrm{MDH}$ and $S c \mathrm{MDH}-\mathrm{T} 4$ require further investigation to support the speculated reasons for coenzyme specificity alternation in ScMDH.

\section{ACKNOWLEDGMENTS}

Research supported by the National High Technology Research and Development Program ("863" Program: \#2012AA02A708), the National Natural Science Foundation of China (\#31170005; \#30870062; \#31300006), the Specialized Research Fund for the Doctoral Program of Higher Education of China (\#20113424110004), the Fund of State Key Laboratory of Genetics Resources and Evolution from Kunming Institute of Zoology (CAS) (\#GREKF11-07), the Anhui Provincial Natural Science Foundation (\#1208085QC52), and the Foundation for Excellent Young Talents in University of Anhui Province (\#2009SQRZ030).

\section{REFERENCES}

Andreadeli A, Platis D, Tishkov V, Popov V, et al. (2008). Structure-guided alteration of coenzyme specificity of formate dehydrogenase by saturation mutagenesis to enable efficient utilization of $\mathrm{NADP}^{+}$. FEBS J. 275: 3859-3869.

Ge YD, Cao ZY, Wang ZD, Chen LL, et al. (2010). Identification and biochemical characterization of a thermostable malate dehydrogenase from the mesophile Streptomyces coelicolor A3(2). Biosci. Biotechnol. Biochem. 74: 21942201.

Goward CR and Nicholls DJ (1994). Malate dehydrogenase: a model for structure, evolution, and catalysis. Protein Sci. 3: $1883-1888$.

Hara S, Motohashi K, Arisaka F, Romano PG, et al. (2006). Thioredoxin-h1 reduces and reactivates the oxidized cytosolic malate dehydrogenase dimer in higher plants. J. Biol. Chem. 281: 32065-32071.

Krahulec S, Klimacek M and Nidetzky B (2012). Analysis and prediction of the physiological effects of altered coenzyme specificity in xylose reductase and xylitol dehydrogenase during xylose fermentation by Saccharomyces cerevisiae. J. Biotechnol. 158: 192-202.

Lee SM, Kim JH, Cho EJ and Youn HD (2009). A nucleocytoplasmic malate dehydrogenase regulates p53 transcriptional activity in response to metabolic stress. Cell Death Differ. 16: 738-748.

Lesk AM (1995). NAD-binding domains of dehydrogenases. Curr. Opin. Struct. Biol. 5: 775-783.

Levy HR, Vought VE, Yin X and Adams MJ (1996). Identification of an arginine residue in the dual coenzyme-specific glucose-6-phosphate dehydrogenase from Leuconostoc mesenteroides that plays a key role in binding NADP ${ }^{+}$but not $\mathrm{NAD}^{+}$. Arch. Biochem. Biophys. 326: 145-151.

Liu Q, Harvey CT, Geng H, Xue C, et al. (2013). Malate dehydrogenase 2 confers docetaxel resistance via regulations of JNK signaling and oxidative metabolism. Prostate 73: 1028-1037.

Madern D, Cai X, Abrahamsen MS and Zhu G (2004). Evolution of Cryptosporidium parvum lactate dehydrogenase from malate dehydrogenase by a very recent event of gene duplication. Mol. Biol. Evol. 21: 489-497.

Menckhoff L, Mielke-Ehret N, Buck F, Vuletić M, et al. (2013). Plasma membrane-associated malate dehydrogenase of maize (Zea mays L.) roots: Native versus recombinant protein. J. Proteomics 80C: 66-77.

Ocheretina O, Haferkamp I, Tellioglu H and Scheibe R (2000). Light-modulated NADP-malate dehydrogenases from mossfern and green algae: insights into evolution of the enzyme's regulation. Gene 258: 147-154.

Rosell A, Valencia E, Ochoa WF, Fita I, et al. (2003). Complete reversal of coenzyme specificity by concerted mutation 
of three consecutive residues in alcohol dehydrogenase. J. Biol. Chem. 278: 40573-40580.

Rossmann MG, Moras D and Olsen KW (1974). Chemical and biological evolution of nucleotide-binding protein. Nature 250: 194-199.

Schepens I, Johansson K, Decottignies P, Gillibert M, et al. (2000). Inhibition of the thioredoxin-dependent activation of the NADP-malate dehydrogenase and cofactor specificity. J. Biol. Chem. 275: 20996-21001.

Schnarrenberger C and Martin W (2002). Evolution of the enzymes of the citric acid cycle and the glyoxylate cycle of higher plants. A case study of endosymbiotic gene transfer. Eur. J. Biochem. 269: 868-883.

Scrutton NS, Berry A and Perham RN (1990). Redesign of the coenzyme specificity of a dehydrogenase by protein engineering. Nature 343: 38-43.

Simionatto S, Marchioro SB, Galli V, Luerce TD, et al. (2009). Efficient site-directed mutagenesis using an overlap extension-PCR method for expressing Mycoplasma hyopneumoniae genes in Escherichia coli. J. Microbiol. Methods 79: 101-105.

Tomita T, Fushinobu S, Kuzuyama T and Nishiyama M (2005). Crystal structure of NAD-dependent malate dehydrogenase complexed with NADP(H). Biochem. Biophys. Res. Commun. 334: 613-618.

Tomita T, Kuzuyama T and Nishiyama M (2006a). Alteration of coenzyme specificity of lactate dehydrogenase from Thermus thermophilus by introducing the loop region of NADP(H)-dependent malate dehydrogenase. Biosci. Biotechnol. Biochem. 70: 2230-2235.

Tomita T, Fushinobu S, Kuzuyama T and Nishiyama M (2006b). Structural basis for the alteration of coenzyme specificity in a malate dehydrogenase mutant. Biochem. Biophys. Res. Commun. 347: 502-508.

Watanabe S, Kodaki T and Makino K (2005). Complete reversal of coenzyme specificity of xylitol dehydrogenase and increase of thermostability by the introduction of structural zinc. J. Biol. Chem. 280: 10340-10349.

Zhu G, Golding GB and Dean AM (2005). The selective cause of an ancient adaptation. Science 307: 1279-1282. 